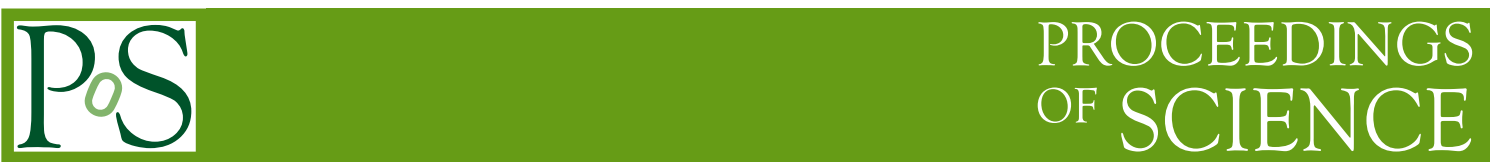

\title{
Infra-red problems and a response
}

\section{Anton Ilderton}

Department of Physics, Umeå University, 90187 Umeå, Sweden

\section{Tom Heinzl, Kurt Langfeld, Martin Lavelle*, David McMullan}

School of Computing and Mathematics, University of Plymouth, PL4 9NG, UK

E-mail: mlavelle@plymouth.ac.uk

\begin{abstract}
The standard approach to the infra-red problem is to sum over degenerate final states to remove soft divergences (Bloch-Nordsiech), and over both initial and final states for collinear divergences (Lee-Nauenberg). We show that this division is inconsistent, and further that the Lee-Nauenberg recipe leads to ill-defined results in a variety of theories. We then argue that infra-red divergences are due to using unphysical variables to describe in and out states and introduce gauge invariant, physical 'dressed' states. Perturbative and non-perturbative properties of physical electron and quark states are then discussed. It is shown in SU(2) lattice simulations that the overlap between the ground state and our dressed state increases as we approach finer lattices.
\end{abstract}

International Workshop on QCD Green's Functions, Confinement and Phenomenology

September 7-11, 2009

ECT Trento, Italy

${ }^{*}$ Speaker. 


\section{Introduction}

In scattering calculations we tend to assume that the coupling constant can be set to zero at infinitely remote times. In QED this might appear reasonable while in QCD such calculations are often based on the premise of some parton-hadron duality. The results of scattering calculations are, though, plagued by infra-red (IR) divergences.

The source of the IR problem has long been known to be due to the interaction not falling off fast enough to allow us to neglect asymptotic interactions, even in QED. The usual responses, due to Bloch-Nordsieck (BN) [1] and Lee-Nauenberg (LN) [2] both, as we describe below, incorporate final (BN) or initial and final (LN) state interactions through soft and collinear emission and absorbtion. In this talk we will show through explicit examples that both these responses to the IR problem fail in gauge theories. As a result we will construct different initial and final states which are gauge invariant and have a physical interpretation. The results of perturbative calculations and nonperturbative lattice simulations support the significance of this construction.

\section{IR and Collinear Divergences}

The process which we consider here is Coulomb scattering. At one loop

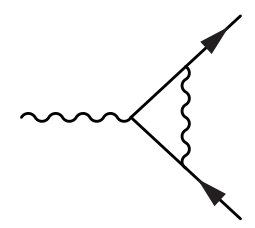

there are UV and IR divergences. The UV and soft IR divergences are regularised by working in $D$ dimensions with, for the IR divergences, $D=4+2 \varepsilon$ and, for the collinear divergences, we retain a small fermion mass, $m \neq 0$.

Coulomb scattering is described in terms of two structure functions. One, $F_{2}$, is IR finite and IR safe (this means it is not affected by the real emission and absorbtion processes used in $\mathrm{BN}$ and in LN). However, the other, $F_{1}$, suffers from both soft and collinear divergences. Schematically there are soft divergences, $\frac{1}{\varepsilon}$, leading and sub-leading collinear divergences, $\ln ^{2}(m)$ and $\ln (m)$, and crossed divergences, $\frac{1}{\varepsilon} \ln (m)$. The gory details can be found in [3]; here we just want to bring out the form of the (non-)cancellations.

The Bloch-Nordsieck response to this problem is to include degenerate real, soft emission, i.e., photons with energy up to a maximum $\Delta$ beyond which the detector in the experiment could distinguish them.

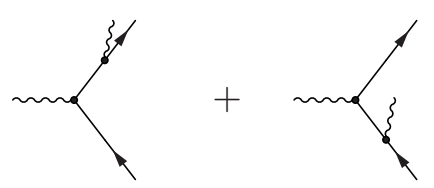

It should be stressed that $\mathrm{BN}$ do not include absorbtion processes. Typically calculations following the BN trick work in the eikonal approximation (dropping soft momenta in the numerator) as this, by power counting, does not affect the soft divergent structures. Emission diagrams like the above are squared and the result added to the virtual processes at the level of the cross-section. 
The BN trick works surprisingly well: all the divergences cancel at the level of such semiinclusive cross-sections except for the sub-leading collinear logarithms. The natural extension of $\mathrm{BN}$ is to include the emission of non-soft (semi-hard) photons which are collinear with the outgoing electron and would thus not be distinguishable processes. Emissions off the outgoing line

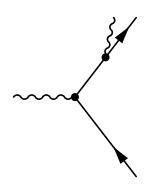

indeed generate collinear logs. However, they only generate half the amount needed to produce a cancellation. This naturally leads to the idea, widespread in the literature, that one should add collinear absorbtion on the incoming leg which will produce, at the level of the cross-section, what we need for a cancellation. This is the essence of the LN paper.

It may well seem rather strange that we are admonished by LN to include semi-hard collinear absorbtion but not soft absorbtion. However, as we will now show, it is actually inconsistent to divide our treatment in this way (soft $\Leftrightarrow$ emission only à la $\mathrm{BN}$; collinear $\Leftrightarrow$ emission and absorbtion à la LN).

A precise treatment [3] shows that semi-hard emission really generates terms of the form

$$
\frac{1}{2} \ln (m) \times\left[\frac{3}{4}-\ln \left(\frac{E}{\Delta}\right)-\frac{\Delta}{E}+\frac{1}{4} \frac{\Delta^{2}}{E^{2}}\right]
$$

The first two terms in the square brackets are of the form needed for the cancellation of the virtual collinear sub-leading logarithms, however, the powers of the resolution $\Delta$ are artefacts of dividing the energy between semi-hard $(E>\Delta$ and soft $(E<\Delta)$ photons. These terms are killed off by considering the sub-eikonal (soft finite) emission terms.

This means that to get a cancellation of all collinear logarithms we would need to define our results through the following semi-inclusive sum: all (soft and hard) emission processes plus those for the absorbtion of semi-hard and of sub-eikonal soft photons. However, the eikonal soft photons cannot be included in the LN framework as they would spoil the cancellation although they come from the same Feynman diagrams and same momentum integration range as the sub-eikonal terms. This is clearly wrong. We need to look for a different way of cancelling the collinear logarithms.

Staying in the spirit of the LN paper, let us see what happens when one adds all indistinguishable degenerate processes including all initial and final state processes. This means including eikonal soft absorbtion processes which of course ruin the BN cancellation. We will therefore now consider massive QED and look at soft divergences in this less-divergent theory in the spirit of LN.

The virtual and degenerate emission processes yield equal and opposite amounts of soft divergences while absorbtion duplicates the emission process. More terms are required for any possible cancellation. We thus now add processes with both emission and absorbtion such as

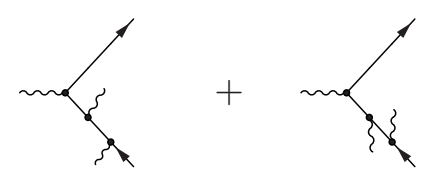


This might seem to only enter the cross-section at higher orders in the coupling, but, as noted in Appendix D of LN's paper, interference with a disconnected photon

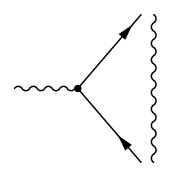

produces connected interference terms in the cross-section at order $e^{4}$. Various authors, see e.g. [4], [5] and [6] have included such processes in the hope of cancelling IR divergences.

The problem with most such work is that it tends to include just enough processes to get a cancellation and then stops. Generally such calculations sum all the above processes plus either an emission or an absorbtion process with a disconnected photon, i.e., squaring up diagrams like

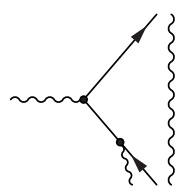

and then retaining the connected contribution at the level of the cross-section, i.e., the left hand but not the right hand terms below
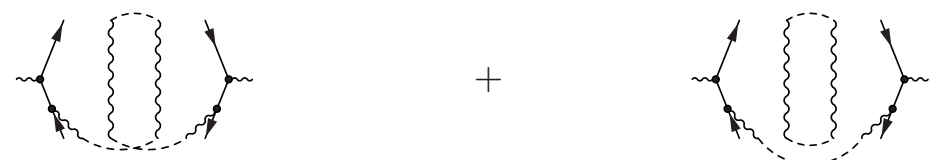

Although this is finite, it is not reasonable and is completely against the spirit of LN who argue one should include all degenerate processes to obtain the result which an experiment would obtain. There is no physical reason to stop at the line above. Why not consider, say, emission plus a disconnected photon or indeed absorbtion with two (or more) disconnected photons, etc. etc.? They will all contribute connected interference terms to the cross-section at order $e^{4}$. There are, in fact, infinitely many possible diagrams at a finite order in the coupling.

In an interesting paper, Ito [7] suggested summing this series of disconnected processes, i.e., at order $e^{4}$ summing all connected contributions to the cross-section from arbitrary numbers of disconnected photons. His result was later rediscovered in [8]. The expression given in these papers is formal, however, if one calculates [3] the value of the coefficients one finds that the series does not converge. It is not that the coefficients fall off slowly, they do not decrease at all! Soft absorbtion S-matrix elements, say, with $1,2,3, \ldots 10, \ldots$ disconnected photons all generate exactly the same soft divergences in the connected cross-section. We are forced to conclude that none of the standard approaches to the IR problem is correct.

Recently [9] we have shown that these breakdowns also reveal themselves when one tries to apply LN to another paradigm example of the IR: $\phi^{3}$ in six dimensions which is collinearly divergent but does not suffer from soft divergences. Again there are infinite towers of diagrams at a fixed order of perturbation theory; the series do not converge and no reliable cancellation could be obtained. Such problems also become yet more severe if one takes into account that initial and final experimental resolutions are likely to differ. 
The lack of cancellations show that a fresh approach is needed. Our starting point is the observation that, as the IR problem is due to interactions falling off slowly, it is not justified to set the coupling asymptotically to zero and so any description of our initial and final states must be locally gauge invariant. (The Lagrangian fermion is, of course, not invariant if the coupling is not set to zero.) Just as the UV problem is due to the use of bare fields, so the IR problem is a consequence of using unphysical fields.

\section{Gauge Invariant Descriptions of Charged States}

The key to our approach is to 'dress' the matter field with the gauge field [11], [12]. This is accomplished by defining

$$
\Psi:=h^{-1}[A] \psi
$$

where local gauge invariance of the dressed field, $\Psi$, follows if

$$
h^{-1}\left[A^{U}\right]=h^{-1}[A] U \quad \text { and } \quad \psi^{U}=U^{-1} \psi .
$$

The immediate questions are: how can we construct dressings, $h^{-1}[A]$, and which ones have a physical interpretation?

A naive response for a fermion-antifermion system might be to link them by a string (path ordered exponential). This is, though, not at all physical. One can immediately calculate the electric potential energy of the state and find [10]

$$
V(x-y) \sim e^{2}|\underline{x}-\underline{y}| \delta^{2}(0),
$$

which is a confining potential (in QED!) with a divergent coefficient! This is really an infinitely excited state and its UV divergences will be investigated below in lattice studies.

A better choice is the locally gauge invariant dressed field

$$
\Psi=\exp \left[-i e \frac{\partial_{i} A_{i}}{\nabla^{2}}\right] \psi
$$

which describes a static electron. The equal time commutator $\left[E_{i}^{a}(x), A_{j}^{b}(y)\right]_{\mathrm{e} t}=i \delta^{a b} \delta(\underline{x}-\underline{y})$ generates the Coulomb field

$$
E_{j} \Psi|0\rangle=-\frac{e}{4 \pi} \frac{r_{j}}{r^{2}} \Psi|0\rangle \text {. }
$$

This may be generalised to moving charges and in a series of papers it has been shown that the on-shell Green's functions and S-matrix elements of these fields are IR finite (soft and phase divergences both cancelling) so long as the on-shell point corresponds to the dressing for that leg, see, e.g., [13], [14].

These constructions can be extended to QCD. The minimal static dressing of QED may be identified as a rotation into Coulomb gauge. In QCD this leads to an algorithm to construct the dressing through a power series in $g$

$$
\exp \left(g \chi^{a} T^{a}\right) \equiv h^{-1}
$$

with $g \chi^{a} T^{a}=\left(g \chi_{1}^{a}+g^{2} \chi_{2}^{a}+g^{3} \chi_{3}^{a}+\cdots\right) T^{a}$ and

$$
\chi_{1}^{a}=\frac{\partial_{j} A_{j}^{a}}{\nabla^{2}} ; \quad \chi_{2}^{a}=f^{a b c} \frac{\partial_{j}}{\nabla^{2}}\left(\chi_{1}^{b} A_{j}^{c}+\frac{1}{2}\left(\partial_{j} \chi_{1}^{b}\right) \chi_{1}^{c}\right)
$$

We will now describe the results of perturbative and lattice studies of this construction. 


\section{The Perturbative Potential and Non-Perturbative Ground State}

In perturbation theory, we have studied the potential energy between such dressed charges. At leading order it is, as one would expect, Coulombic:

$$
V(r)=-\frac{g^{2} C_{F}}{4 \pi r}
$$

At NLO one finds from minimally dressed Coulombic charges [15]

$$
V(r)=-\frac{g^{2} C_{F}}{4 \pi r}\left[1+\frac{g^{2}}{4 \pi} \frac{C_{A}}{2 \pi} \times 4 \log (\mu r)\right]
$$

which, cf. the one-loop beta function

$$
\beta(g)=-\frac{g^{3}}{(4 \pi)^{2}}\left[4-\frac{1}{3}\right]
$$

shows it generates the dominant antiscreening contribution which is known to come from longitudinal glue (minimal dressing). Screening effects are generated [16] by a separately gauge invariant dressing. These perturbative calculations have been extended [17] to NNLO at leading order in $n_{f}$ and it was shown that the results are multiplicatively renormalisable with non-local divergences cancelling. This last was a non-trivial check.

The above perturbative calculations have now been extended to lattices [18], [19] [20]. In this work we have focussed on the overlaps of trial (dressed) states with the true ground state. For some (gauge invariant) trial quark anti-quark state, with the fermion separation $r$, we have at large $T$ :

$$
\left\langle\operatorname{trial}\left|e^{-H T}\right| \operatorname{trial}\right\rangle=|\langle\operatorname{trial} \mid \Omega\rangle|^{2} e^{-V(r) T} .
$$

We extract the overlap $|\langle\operatorname{trial} \mid \Omega\rangle|^{2}$ of our trial state with the lattice ground state $|\Omega\rangle$. Those simulations described below were for SU(2) Yang-Mills on $20^{4}$ lattices using both Wilson and improved actions. The results, as we shall see, display a dramatic dependence on the trial state. For full details, see [20].

In the first trial state we consider, local gauge invariance is achieved by linking the fermions by a (straight) path-ordered exponential. This stringy state is denoted by $|\chi\rangle$ in the diagram below. 


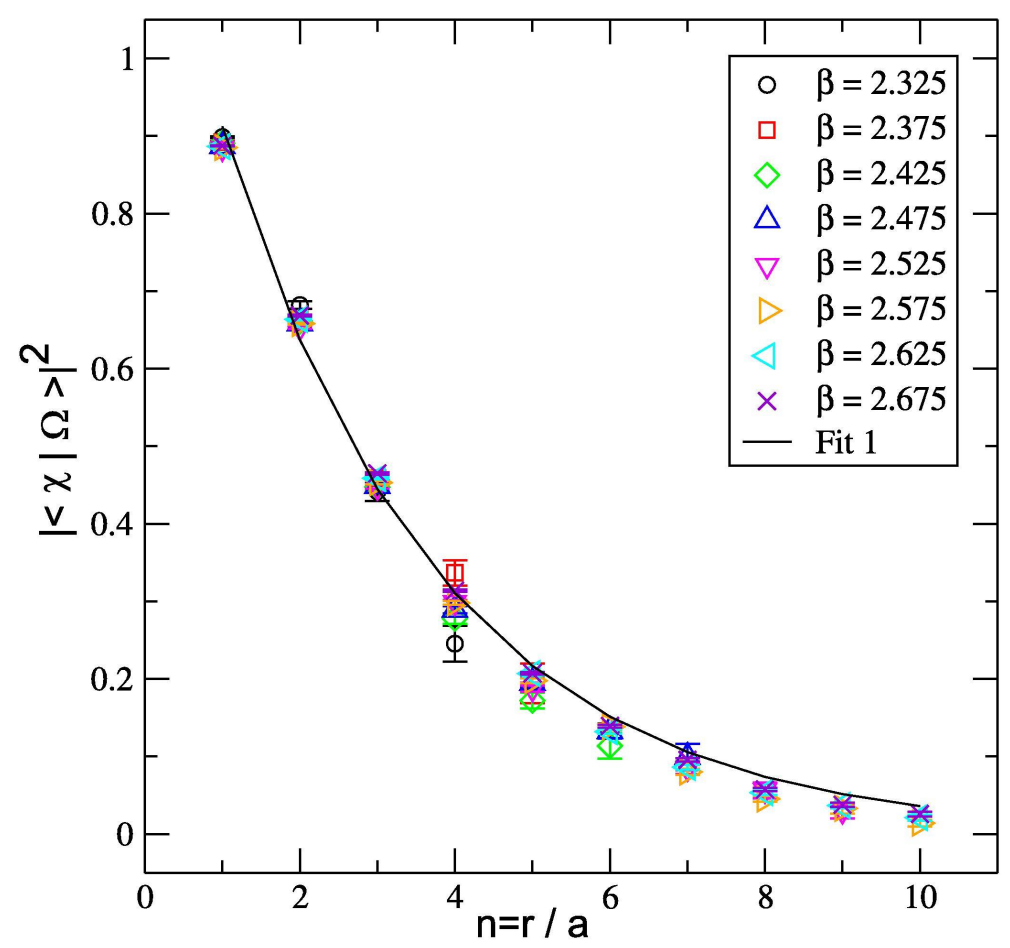

It can be seen that the overlap of the trial state drops exponentially as $n$ increases. This agrees with an analytic, perturbative calculation in QED where the overlap drops exponentially as the UV cut-off is removed. Our interpretation of this is that the continuum limit is highly sensitive to string UV artefacts of the form discussed earlier.

This we argue is the reason why calculations of the potential usually now use smeared states: by broadening the state the UV artefacts of unphysical, infinitely (in the continuum limit) excited states are made less harmful. The results of calculations of the overlap between smeared states, $\left|\chi_{S}\right\rangle$, and the ground state are shown below.

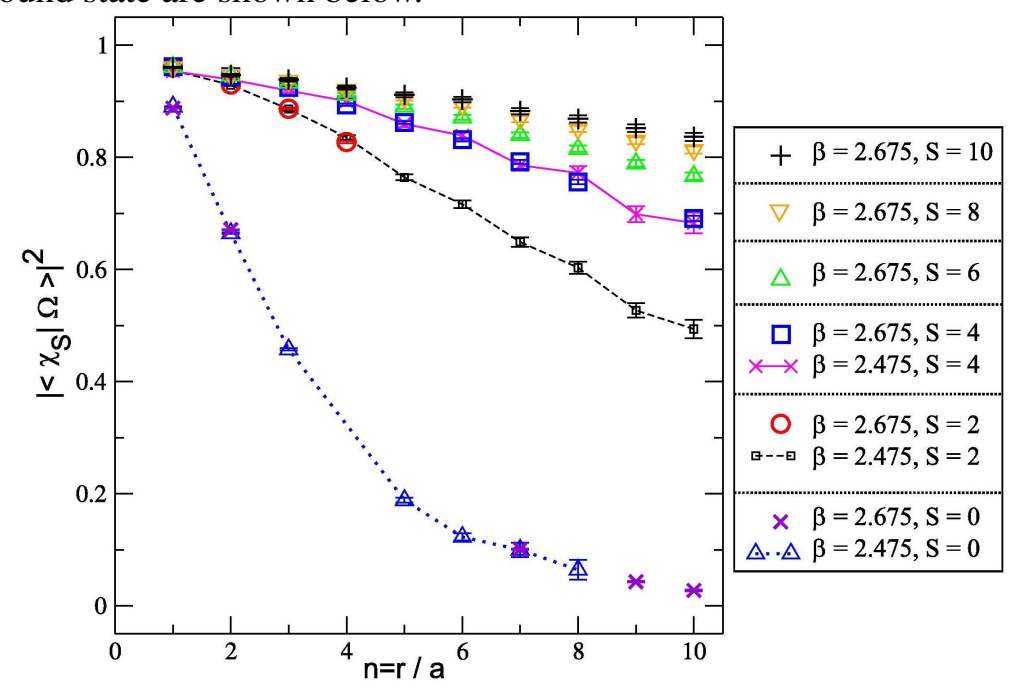

Here the lines joining the points are just to guide the eye. It is again the case that as we go to finer lattices the overlap for any fixed level of smearing decreases. Of course a state which was six, say, lattice spacings wide would still have infinitesimal width in the continuum limit. Thus any finite 
level of smearing will be sensitive to UV artefacts if one goes to fine enough lattices. We predict that future work using smeared states on very fine lattices will require highly smeared states to maintain an acceptable overlap with the ground state.

The next graph shows the results of calculations for overlaps between the ground state and the Coulombic dressed state, $|\Phi\rangle$, which was used in the perturbative calculations mentioned above. The results show a completely different behaviour in the continuum limit:

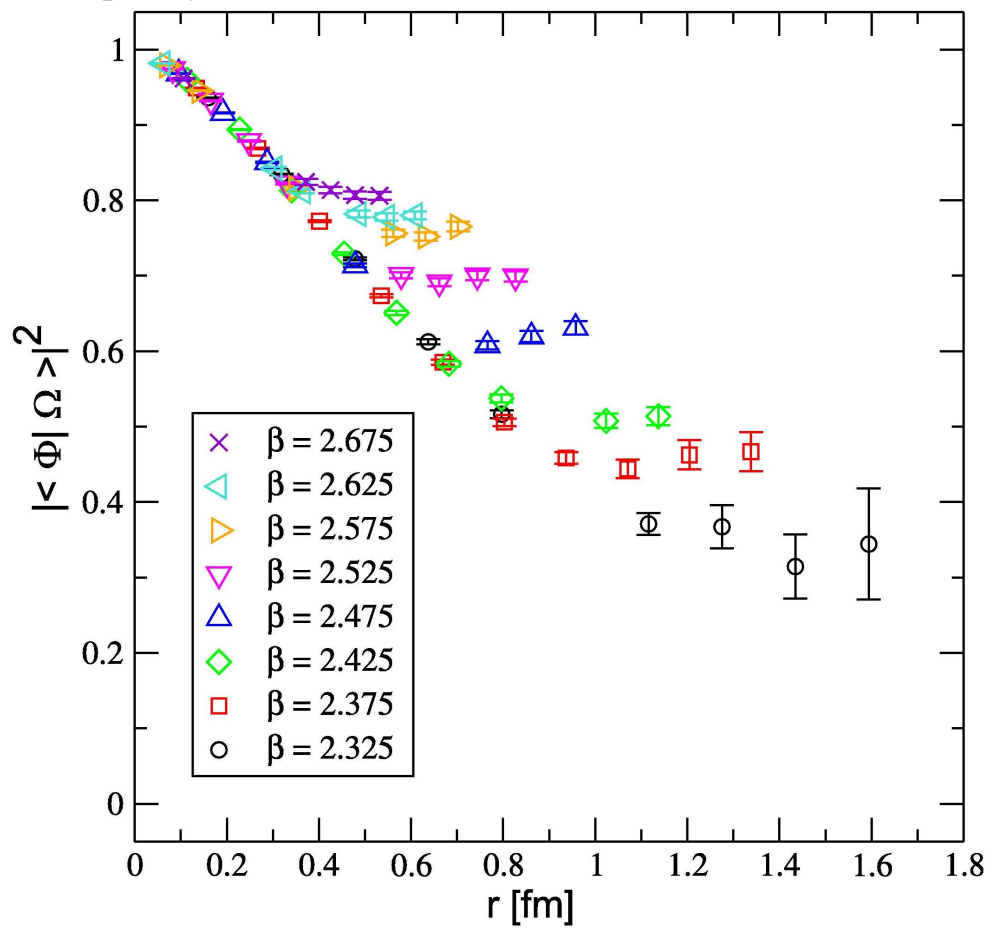

As one goes to finer lattices the Coulombic state has a better overlap with the true ground state. It is not excluded that it is the true ground state in the continuum limit, but we suspect that it is more likely the case that it plays an important part in the ground state but additional, separately gauge invariant, glue is required in the ground state to produce screening effects.

\section{Conclusion}

The first results described above show that in a theory with soft and collinear divergences the standard procedure of using $\mathrm{BN}$ to handle soft divergences and $\mathrm{LN}$ for collinear ones does not work. Trying to use $\mathrm{LN}$ for all divergences leads to a somewhat shocking result: the $\mathrm{LN}$ approach fails in a variety of theories. Most work in the literature simply adds enough processes until IR divergences cancel. Other processes can, however, be added at the same order of perturbation theory due to disconnected diagrams producing connected contributions at the level of the cross-section, as was first pointed out in the initial LN paper. There have been a limited number of attempts to sum all these possible processes at the same order of perturbation theory. However, the sum does not converge [3].

This calls out for a fundamental reappraisal of how to deal with the IR problem. In the rest of the talk we have described an approach based on using locally gauge invariant 'dressed' fields in the initial and final states. Such dressed on-shell Green's functions and S-matrix elements have 
been shown to be multiplicatively renormalisable in the UV and also free of IR divergences. There remains the question of IR safety, i.e., the incorporation of soft emission and absorbtion with such fields. This deserves further study.

Next, we have shown that perturbative and non-perturbative calculations of the inter-quark potential support the relevance of the physical states suggested here. The increase in the overlap of these Coulombic states with the true ground state as one approaches the continuum limit differs qualitatively from the decrease in the overlap of stringy and smeared states with the ground state for finer meshed lattices. This insight into the nature of the QCD ground state deserves further study.

Finally, in the talk by Anton Ilderton it will be shown how to construct a dressed state with zero net charge which is confined to a finite volume [21]. This talk will also address the role of the Gribov ambiguity in obstructing the construction of physical states with well-defined colour charge.

\section{Acknowledgments}

It is a pleasure to thank the organisers for the invitation to participate in this meeting.

\section{References}

[1] F. Bloch and A. Nordsieck, Phys. Rev. 52 (1937) 54.

[2] T.D. Lee and M. Nauenberg, Phys. Rev. 133 (1964) B1549.

[3] M. Lavelle and D. McMullan, JHEP 03 (2006) 026; hep-ph/0511314.

[4] C. De Calan and G. Valent, Nucl. Phys. B42 (1972) 268.

[5] T. Muta and C.A. Nelson, Phys. Rev. D25 (1982) 2222.

[6] A. Axelrod and C.A. Nelson, Phys. Rev. D32 (1985) 2385.

[7] I. Ito, Prog. Theor. Phys. 65 (1982) 1466.

[8] R. Akhoury, M.G. Sotiropoulos and V. Zakharov, Phys. Rev. D56 (1997) 377; hep-ph/9702270.

[9] M.J.Lavelle, D. McMullan and T.G. Steele, in preparation.

[10] P. Haagensen and K. Johnson, On the Wave Functional for Two Heavy Color Sources in Yang-Mills Theory, (1997), hep-th/9702204.

[11] P. A. M. Dirac, Can. J. Phys. 33, 650 (1955).

[12] M. Lavelle and D. McMullan, Phys. Rept. 279 (1997) 1 [arXiv:hep-ph/9509344].

[13] E. Bagan, M. Lavelle and D. McMullan, Annals Phys. 282 (2000) 471 [arXiv:hep-ph/9909257].

[14] E. Bagan, M. Lavelle and D. McMullan, Annals Phys. 282 (2000) 503 [arXiv:hep-ph/9909262].

[15] M. Lavelle and D. McMullan, Phys. Lett. B 436, 339 (1998) [arXiv:hep-th/9805013].

[16] E. Bagan, M. Lavelle, D. McMullan and S. Tanimura, Phys. Rev. D 65, 105004 (2002) [arXiv:hep-ph/0107303].

[17] E. Bagan, M. Lavelle and D. McMullan, Phys. Lett. B 632, 652 (2006) [arXiv:hep-th/0510077]. 
[18] T. Heinzl, K. Langfeld, M. Lavelle and D. McMullan, Phys. Rev. D 76 (2007) 114510 [arXiv:0705.2718 [hep-lat]].

[19] T. Heinzl, A. Ilderton, K. Langfeld, M. Lavelle, W. Lutz and D. McMullan, Phys. Rev. D 77 (2008) 054501 [arXiv:0709.3486 [hep-lat]].

[20] T. Heinzl, A. Ilderton, K. Langfeld, M. Lavelle, W. Lutz and D. McMullan, Phys. Rev. D 78 (2008) 034504 [arXiv:0806.1187 [hep-lat]].

[21] A. Ilderton, M. Lavelle and D. McMullan, [arXiv:0907.4071 [hep-th]]. 